\title{
Nuevos datos de estimación de edad y estación de muerte de pinnípedos en la región del canal Beagle, Tierra del Fuego (Argentina)
}

\author{
María Paz Martinoli, Enrique A. Crespo, Natalia A. Dellabianca \\ y Atilio Francisco J. Zangrando \\ Recibido 09 de abril 2021. Aceptado 05 de agosto 2021
}

\begin{abstract}
RESUMEN
Durante varias décadas, las interpretaciones sobre las pautas de explotación de pinnípedos en el canal Beagle se basaron sobre los conjuntos zooarqueológicos recuperados en el Segundo Componente de Túnel I. En los últimos años, el análisis sistemático de un número mayor de contextos distribuidos en distintos sectores de la costa norte del canal Beagle generó información para momentos más recientes de la secuencia de ocupación. Si bien se mantiene una tendencia en la explotación de machos adultos y subadultos de Arctocephalus australis durante el Holoceno medio, se observa heterogeneidad en el tipo de presas explotadas en el Holoceno tardío en relación con los perfiles de sexo y edad. En este trabajo se presentan y discuten los resultados de cortes delgados efectuados en 15 caninos de pinnípedos a fin de ampliar estimaciones sobre edad y estación de muerte de especímenes recuperados en contextos del Holoceno tardío en el canal Beagle. Los resultados indican variabilidad en los perfiles de edad respecto de los determinados previamente y capturas de pinnípedos en todas las estaciones del año durante el Holoceno tardío. Se concluye que es posible que las estrategias de aprovechamiento se hayan diversificado hacia momentos tardíos en la historia de ocupaciones de dicha región.
\end{abstract}

Palabras clave: Estacionalidad de muerte; Pinnípedos; Zooarqueología; Canal Beagle.

\section{New data on age and death seasonality estimations of pinnipeds in the Beagle channel, Tierra del Fuego (Argentina)}

\begin{abstract}
For decades, studies of past pinniped exploitation in the Beagle Channel were mainly based on the zooarchaeological assemblages in the Second Component of Túnel I site, dated to the middle Holocene. In recent years, the systematic analysis of further contexts located at different sectors of the north coast of the Beagle Channel have provided new information, especially for the late Holocene. While a hunting pattern focused on adult and subadult males of $A$. australis remain apparent in middle Holocene assemblages, age and sex profiles show a more heterogeneous pattern in later moments of the archaeological sequence. In this paper, new estimations on the age and season of death of pinniped specimens recovered from archaeological contexts in the Beagle Channel are presented. The results,

María Paz Martinoli. Centro Austral de Investigaciones Científicas (CADIC)-Consejo Nacional de Investigaciones Científicas y Técnicas (CONICET). Bernardo Houssay 200 (CP 94I0), Ushuaia, Tierra del Fuego, Argentina. E-mail: mpmartinoli@yahoo.com.ar Enrique A. Crespo. Centro para el Estudio de Sistemas Marinos (CESIMAR)-CONICET. Boulevard Brown 2915 (CP 9120), Puerto Madryn, Chubut, Argentina. E-mail: crespocnp@gmail.com

Natalia A. Dellabianca. Laboratorio de Ecología y Conservación de Vida Silvestre CADIC-CONICET. Bernardo Houssay 200 (CP 94I0), Ushuaia, Tierra del Fuego, Argentina. Fundación R. Natalie P. Goodall, Museo Acatushún de Aves y Mamíferos Marinos Australes, Ea. Harberton, Tierra del Fuego, Argentina. E-mail: ndellabianc@gmail.com

Atilio Francisco J. Zangrando. CADIC-CONICET. Bernardo Houssay 200 (CP 94I0), Ushuaia, Tierra del Fuego, Argentina. E-mail: panchozan@yahoo.com.ar
\end{abstract}

Intersecciones en Antropología 22(2), julio-diciembre: 195-207. 2021. ISSN-e 1850-373X

https://doi.org/10.37176/iea.22.2.2021.629

Facultad de Ciencias Sociales - UNICEN - Argentina 
based on thin sections of canine teeth, indicate variation from the age profiles previously obtained from zooarchaeological studies. The results also indicate a seasonally diverse pattern in pinniped capture during the late Holocene. It is argued that pinniped harvesting strategies probably varied in the Beagle Channel throughout the Holocene.

Keywords: Death seasonality; Pinnipeds; Zooarchaeology; Beagle Channel.

\section{INTRODUCCIÓN}

Los pinnípedos constituyeron un recurso clave para las poblaciones humanas que habitaron el canal Beagle a partir del Holoceno medio (Orquera y Piana, 1987, 1999, 2009; Schiavini, 1990, 1993; Zangrando, 2009; Zangrando et al., 2014; Martinoli, 2018; Nye et al., 2020). Estas presas no solo aportaron nutrientes con elevados valores energéticos, sino que fueron fuente de materias primas para la producción de tecnología (Orquera y Piana, 1987, 1999, 2009; Scheinsohn y Ferretti, 1995; Scheinsohn, 1997, 2010; Christensen, 2016). La variabilidad en la explotación de tales mamíferos marinos puede ser analizada en el registro arqueológico a través de diferentes escalas que incluyen consideraciones biogeográficas en la distribución de colonias reproductivas y movimientos estacionales de estos organismos (Lyman, 1989, 2003; Schiavini, 1993; Borella, 2006), además de las variaciones conductuales detectadas a lo largo de siglos o milenios (Newsome et al., 2010; Zangrando et al., 2014; Vales et al., 2017; Nye et al., 2020). Para un examen paleoecológico de estos factores resultan necesarias las determinaciones de especie, sexo, edad y estación de muerte de los especímenes arqueológicos (Lyman, 1989; Schiavini, 1990; Borella, 2006, 2016; Muñoz, 2011; Martinoli, 2015, 2017, 2018).

Los primeros estudios zooarqueológicos sobre restos de pinnípedos recuperados en el Segundo Componente de Túnel I (Holoceno medio; 60004000 años AP), así como el análisis de muestras más recientes procedentes de niveles de conchero de Imiwaia I (6000 años AP), indicaron que las capturas se efectuaron principalmente sobre machos adultos e individuos subadultos de sexo indeterminado de lobo marino de dos pelos (Arctocephalus australis), con un bajo porcentaje de especímenes de lobo marino de un pelo (Otaria flavescens) (Schiavini, 1990, 1993; Orquera y Piana, 1999; Martinoli, 2013, 2015, 2018). A su vez, a partir de la lectura de las capas de crecimiento en caninos del Segundo Componente de Túnel I, se observó que las muertes ocurrieron mayormente en otoño e invierno (Schiavini, 1990). Los perfiles de mortalidad, en conjunto con los datos de estacionalidad, Ilevaron a proponer que las capturas habrían ocurrido fuera del período reproductivo, momento en el cual machos adultos y subadultos realizan viajes de alimentación (Schiavini, 1990, 1993; Orquera y Piana, 1999). La propuesta se basaba en la distribución actual de estos mamíferos en la región: los espacios reproductivos de $A$. australis están concentrados en sectores exteriores del archipiélago fueguino (Schiavini, 1990; Schiavini y Raya Rey, 2001; Milano et al., 2020). Dicho modelo de aprovisionamiento sostenía, por un lado, que los ejemplares que se dispersaban desde estos espacios hacia los canales interiores aportaban a los grupos humanos un subsidio energético (Schiavini, 1990, 1993). Por otro lado, que este modo de captura -sin impactar de manera directa los espacios reproductivos- habría permitido evitar una explotación intensiva del recurso durante el Holoceno (Orquera y Piana, 1999).

Evaluaciones zooarqueológicas en las que se analizó toda la secuencia de la región del canal Beagle señalaron variaciones a largo plazo en la explotación de recursos animales, entre las cuales se identificó una disminución en la representación de pinnípedos luego de 5000-4000 años AP y una intensificación en la explotación de aves y peces para momentos más tardíos de la secuencia (Zangrando, 2009; Tivoli y Zangrando, 2011). Asimismo, estudios recientes de los perfiles de mortalidad para conjuntos arqueológicos de otáridos, con distintas cronologías y considerando criterios anatómicos poscraneales (ver criterios en: Pérez García, 2003, 2008; Cadegán Sepúlveda, 2013; Borella et al., 2018; Martinoli, 2018), muestran un aumento relativo en la presencia de hembras en edad reproductiva y de individuos menores a un año (cachorros) de $A$. australis, especialmente en el Holoceno tardío (Saxon, 1979; Estévez y Martínez, 1995; Orquera y Piana, 1995; Zangrando, 2014; Martinoli, 2017, 2018; Martinoli y Vázquez, 2017; Martinoli y Zangrando, 2021). Es decir que es posible pensar que hubo variaciones en las actividades de aprovisionamiento de pinnípedos hacia momentos finales del Holoceno. 
Como se señaló previamente, con el estudio de la muestra recuperada en el Segundo Componente de Túnel I (Holoceno medio) se estableció que la mayoría de las presas fueron capturadas en otoñoinvierno (Schiavini, 1990). No obstante, al considerar la información indicada más arriba, también es posible esperar variaciones en la estacionalidad de las capturas en momentos posteriores de la secuencia arqueológica, dado un aumento relativo en la presencia de categorías de edad y sexo propias de espacios reproductivos, los que se forman en verano (Schiavini y Raya Rey, 2001; Schiavini et al., 2004; Milano et al., 2020). El propósito de este trabajo es contribuir a esta discusión mediante el análisis de perfiles de edad y estación de muerte en conjuntos de pinnípedos del canal Beagle. Se presentan los resultados obtenidos de la lectura de bandas de crecimiento en caninos recuperados en Imiwaia I (capas K, L y M; $5943 \pm 48$ años AP),
Ajej I (capa C; $1400 \pm 90$ años AP) y Kaiyawoteha II (capas L, M, Ñ y T; $730 \pm 45$ años AP) (Figura 1). Así, mediante esta información y los antecedentes disponibles, se integran datos para una diversidad mayor de contextos del canal Beagle.

\section{MATERIALES Y MÉTODOS}

El análisis se efectuó sobre 15 caninos de pinnípedos procedentes de tres sitios arqueológicos (Tabla 1). Se consideraron diversos criterios para la determinación de los individuos muestreados (lateralidad, determinación de la especie, la edad y el sexo). En el caso de especímenes en los que las características eran coincidentes, se utilizó un único elemento de igual lateralidad (Grayson, 1984; Lyman, 1994). Se consideraron únicamente los caninos completos, tanto los hallados de manera aislada

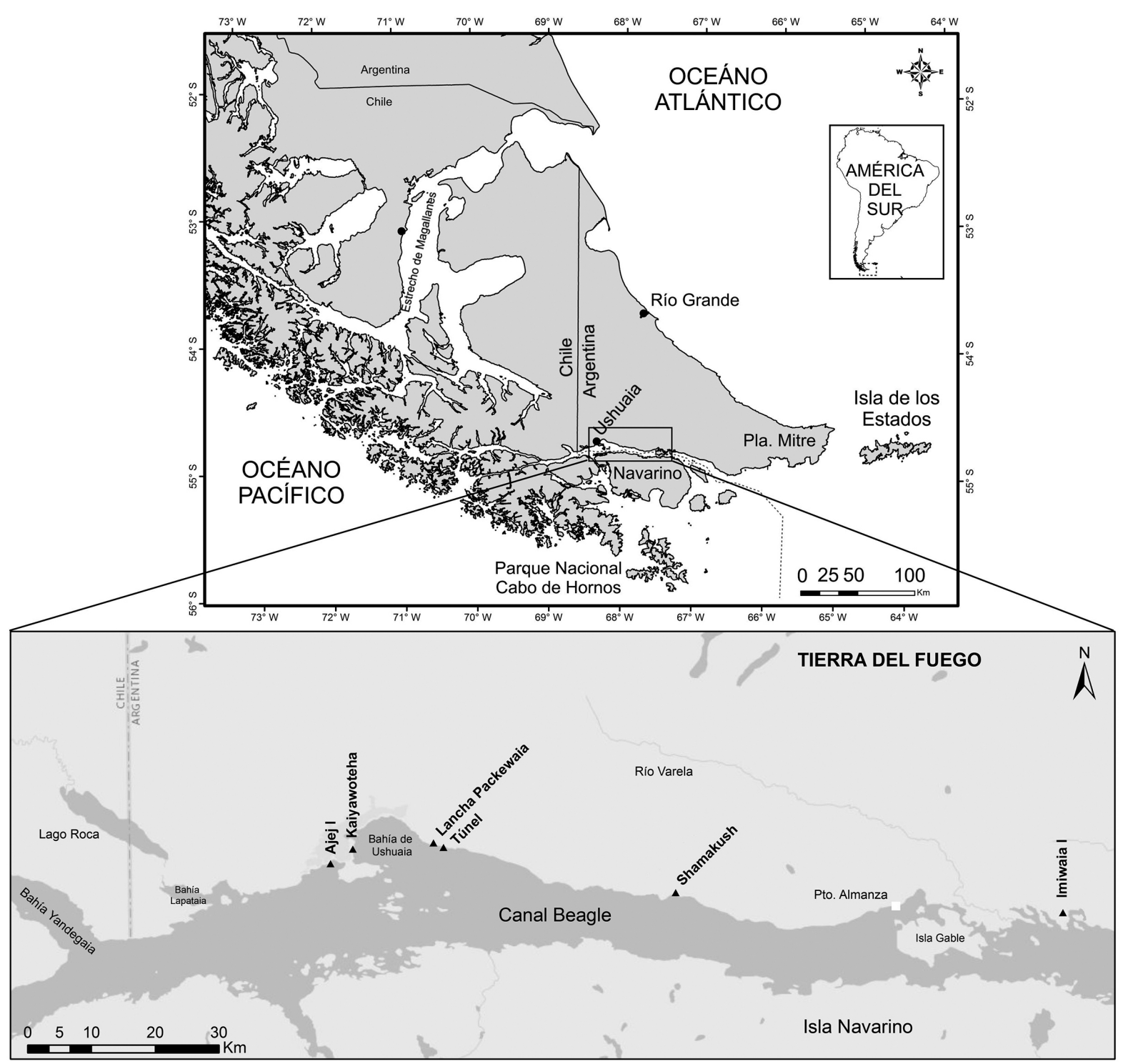

Figura 1. Mapa de la región central del canal Beagle, con indicación de los sitios analizados. 
como los articulados con los maxilares (detalles en Tabla 1).

En el conjunto correspondiente al Holoceno medio de Imiwaia I se hallaron 29 caninos, a partir de los cuales se contabilizaron seis individuos. Cinco de los caninos fueron cortados para el análisis de estacionalidad de muerte. El NMI establecido para este conjunto a partir de elementos poscraneales fue de 36. Con respecto a las muestras con cronologías del Holoceno tardío, en Ajej I se recuperaron 21 caninos, en función de los cuales se identificaron ocho individuos. Seis de estos elementos fueron cortados. El NMI identificado a partir de elementos poscraneales fue de 11. En Kaiyawoteha II, los elementos correspondientes a caninos son 50, el $\mathrm{NMI}$ es siete y se cortaron cuatro caninos; en este último caso, el NMI establecido a partir de elementos poscraneales ascendió a 40. En primer lugar, se destaca la discrepancia entre el NMI total determinado a partir de los huesos del poscráneo y de los caninos, especialmente en Imiwaia I y en Kaiyawoteha II, en cuyo caso los individuos determinados por los caninos no superan el $20 \%$ del NMI total (Tabla 1). Como indican los estudios previos, se observa una presencia relativamente baja de las unidades anatómicas correspondientes al cráneo en estos conjuntos, lo que fue interpretado como parte de una estrategia de procesamiento tendiente al descarte de la cabeza por ser una porción de poco valor económico (Martinoli, 2013, 2018). En segundo lugar, no fue posible realizar cortes delgados en todos los individuos determinados por los caninos, ya que si bien la mayoría de estos elementos estaban completos, algunos de ellos presentaban alteraciones como pérdida de capas de esmalte, agrietamientos longitudinales y alteración térmica, lo que podía indicar que la estructura del diente se encontraba comprometida. Por estos motivos, se seleccionaron para su corte aquellos especímenes cuyo aspecto exterior denotaba buena preservación. A continuación se detallan los criterios de selección y discriminación.

La selección de caninos para llevar adelante este estudio responde a diferentes razones. En primer lugar, análisis comparativos especializados indican que dicho elemento es el más adecuado para realizar el conteo de anillos de crecimiento en otáridos (Loza et al., 2016). En segundo lugar, estas piezas dentarias tienen características morfológicas que permiten la determinación de lateralidad, y su pertenencia al maxilar o a la mandíbula. Estos rasgos son observables a partir de la curvatura anteriormente convexa que presentan (Schiavini, 1990). En tercer lugar, los caninos permiten realizar la determinación taxonómica específica, por su morfología. En el caso de los dientes ubicados en los maxilares, se utilizaron rasgos del cráneo (Legoupil, 1989) y se compararon con colecciones de referencia (Museo Acatushún y Centro Austral

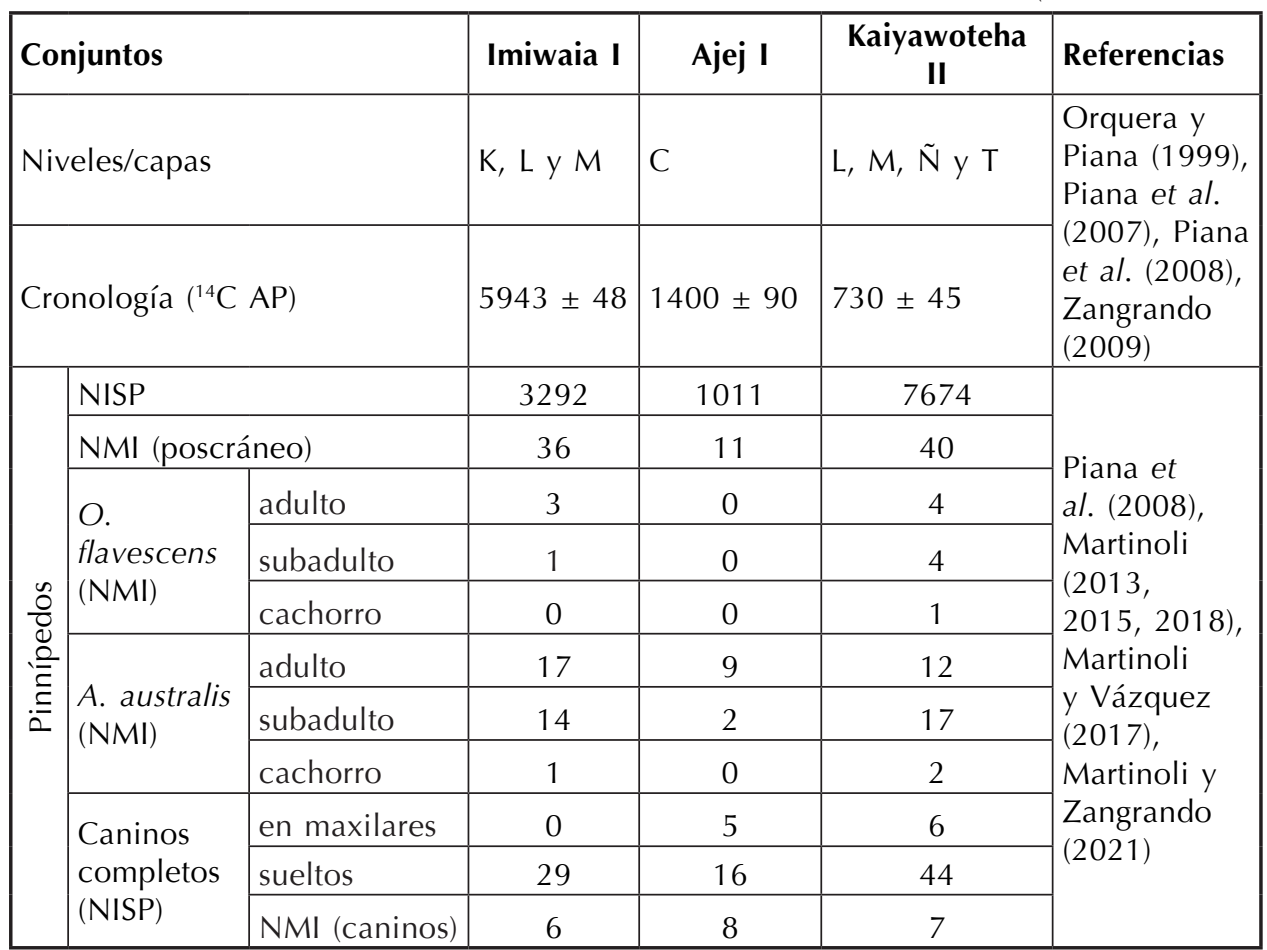

Tabla 1. Información de procedencia y contextual de los conjuntos de pinnípedos analizados en la región del canal Beagle. Detalle de los caninos que conforman los conjuntos faunísticos. de Investigaciones Científicas: C A D I C CONICET). Se consideraron: a) la forma del hueso palatino; b) la forma de la arcada dentaria superior; y c) la forma de la mandíbula (Legoupil, 1989). En el caso de los dientes que se encontraban sueltos, se utilizaron las diferencias alveolares entre ambas especies (Sanfelice y Feroglio, 2008) y el desarrollo de los anillos 
exteriores (Schiavini, 1990). En cuarto lugar, se cuenta con criterios métricos para la determinación del sexo a partir de caninos. En $A$. australis, se midió el ancho máximo de la corona de los caninos: valores menores/iguales a $8 \mathrm{~mm}$ corresponden a hembras, mientras que medidas mayores corresponden a machos, independientemente de la edad de los individuos (Schiavini, 1990, p. 280: Figura 28). Para O. flavescens, el ancho mandibular (amplitud de la mandíbula a nivel de los caninos mandibulares) (Crespo, 1984, p. 248: Figura 3) presenta diferencias entre machos y hembras, con una media de $26,2 \mathrm{~mm}( \pm 8,9 \mathrm{~mm})$ para los primeros y de 15,6 $\mathrm{mm}( \pm 3,1 \mathrm{~mm})$ para las segundas (Crespo, 1984, 1988). Las medidas fueron tomadas con un calibre digital, con rango de medición de 0-300 mm y una resolución de 0,01 $\mathrm{mm}$.

Para los cortes delgados, los dientes se prepararon siguiendo el método descrito en Myrick y colaboradores (1983) y Hohn y colaboradores (1989). Se obtuvieron secciones medias de 2-3 mm de los caninos con una sierra de precisión (LECO VC-50). Posteriormente, se colocaron en formalina al $10 \%$ por más de $3 \mathrm{~h}$, se enjuagaron con agua y luego se descalcificaron con RDO (mezcla comercial de ácidos, Apex Engineering Products Corporation). El tiempo de descalcificación varió entre 3 y 6 h. Los dientes descalcificados y enjuagados se cortaron en secciones delgadas longitudinales de $25 \mu \mathrm{m}$ de espesor con un micrótomo de congelamiento. Las secciones de los dientes se obtuvieron en una orientación bucal-lingual (BL), se tiñeron con hematoxilina de Mayer, se "azularon" en una solución débil de amoniaco y se enjuagaron con abundante agua. Posteriormente, se las incluyó en glicerina al 50\% durante media hora y en glicerina al 100\% hasta el momento del montaje. Por último, se seleccionaron las mejores secciones y se montaron en un portaobjetos en glicerina al $100 \%$.

Para estimar la edad a partir de los dientes se hicieron dos lecturas con microscopio estereoscópico teniendo en cuenta que la erupción dentaria de pinnípedos se origina durante el primer año de vida (Crespo, 1988; Schiavini et al., 1992). De esta forma se puede conocer la edad a través del estudio del incremento de la estructura del diente por medio del conteo de bandas de dentina (Crespo et al., 1994). La estación de muerte en otáridos se determina sobre la base de que la banda clara o traslúcida se forma en la temporada de cría (siempre que el diente se observe con luz transmitida) (Schiavini, 1990); esto es, entre fines de diciembre y fines de enero. El resto del año se deposita la banda opaca, que alcanza su desarrollo final hacia la época de reproducción; ambas conformarían un grupo de líneas de crecimiento (GLC) anual (Perrin y Myrick, 1980; Schiavini et al., 1992; Rosas et al., 1993; Molina-Schiller y Pinedo, 2004). En trabajos previos (e.g., Schiavini, 1990; Ambrústolo et al., 2019), la información de la estación de muerte fue presentada con resolución mensual, considerando las microláminas que se forman de acuerdo con los ciclos lunares. Sin embargo, estudios especializados indican que en el caso de $A$. australis, tales líneas incrementales son conspicuas solo en los primeros tres GLC (Molina-Schiller y Pinedo, 2004). Por este motivo, en el presente trabajo la estación de muerte se determinó como la proporción de capa opaca depositada junto a la última capa translúcida y la cavidad pulpar, y se utilizaron como referencia las estaciones del año.

Como complemento de los análisis mencionados, se realizaron lecturas de los anillos externos presentes en los caninos. Si bien este método es habitualmente utilizado, es necesario realizar algunas aclaraciones (Molina-Schiller y Pinedo, 2004): a) los anillos externos ofrecen una lectura segura solo hasta el séptimo; b) la identificación del primer anillo puede ser compleja en algunos casos; y c) no todos los individuos presentan anillos de crecimiento externos, especialmente las hembras. Por lo dicho, la lectura de los anillos externos puede discrepar con respecto a la lectura de las estructuras de crecimiento a partir de cortes delgados descalcificados y teñidos; este último método es el más adecuado para determinar la edad en otáridos (Molina-Schiller y Pinedo, 2004).

Por último, para la determinación etaria se utilizaron tres categorías: cachorros (menos de un año), subadultos (entre uno y siete años) y adultos (más de siete años). Esta clasificación responde a dos aspectos: los cambios en el comportamiento relacionados con la alimentación y la maduración sexual (Lyman, 1988) y el esquema de fusión del esqueleto poscraneal (Pérez García, 2008; Borella et al., 2013).

\section{RESULTADOS}

A partir del análisis de los caninos se destaca cierta variabilidad, tanto en los perfiles de mortalidad como en la estación de muerte entre los distintos conjuntos (Tabla 2). En el caso de Imiwaia 


\begin{tabular}{|l|l|l|l|l|l|l|l|l|}
\hline Sitio & $\begin{array}{c}\text { Unidad } \\
\text { Anatómica }\end{array}$ & Lat. & $\begin{array}{c}\text { Especie } \\
\text { según rasgos } \\
\text { morfológicos }\end{array}$ & $\begin{array}{c}\text { Ancho } \\
\text { corona }\end{array}$ & \multicolumn{1}{|c|}{ Sexo } & $\begin{array}{c}\text { Edad } \\
\text { (anillos } \\
\text { externos) }\end{array}$ & $\begin{array}{c}\text { Edad (cortes } \\
\text { delgados) }\end{array}$ & Estacionalidad \\
\hline Imi I & Canino superior & Izq. & A. australis & $12 \mathrm{~mm}$ & Macho & 7 años & Mala conserv. & Mala conserv. \\
\hline Imi I & Canino superior & Der. & A. australis & $11 \mathrm{~mm}$ & Macho & 1 año & Mala conserv. & Mala conserv. \\
\hline Imi I & Canino superior & Izq. & A. australis & $10 \mathrm{~mm}$ & Macho & 3 años & Mala conserv. & Mala conserv. \\
\hline Imi I & Canino superior & Der. & A. australis & $12 \mathrm{~mm}$ & Macho & 1 año & Mala conserv. & Mala conserv. \\
\hline Imi I & Canino superior & Der. & A. australis & $11 \mathrm{~mm}$ & Macho & 1 año & Mala conserv. & Mala conserv. \\
\hline Ajej I & Canino inferior & Der. & A. australis & $11 \mathrm{~mm}$ & Macho & 5 años & 4 años & - \\
\hline Ajej I & Canino inferior & Der. & A. australis & $6 \mathrm{~mm}$ & Hembra & - & 6 años & Otoño \\
\hline Ajej I & Canino inferior & Der. & A. australis & $11 \mathrm{~mm}$ & Macho & 3 años & 5 años & Otoño \\
\hline Ajej I & Canino inferior & Der. & A. australis & $7 \mathrm{~mm}$ & Hembra & - & 4 años & Verano \\
\hline Ajej I & Canino inferior & Der. & A. australis & $6 \mathrm{~mm}$ & Hembra & - & 5 años & Verano \\
\hline Ajej I & Canino inferior & Der. & A. australis & $7 \mathrm{~mm}$ & Hembra & 6 años & 7 años & Verano \\
\hline Kaiya II & - & Der. & indet & indet & - & 5 años & 6 años & Verano \\
\hline Kaiya II & Canino superior & Izq. & A. australis & $9 \mathrm{~mm}$ & Macho & 10 años & 8 años & Verano \\
\hline Kaiya II & Canino superior & Izq. & A. australis & $12 \mathrm{~mm}$ & Macho & 8 años & 5 años & Otoño \\
\hline Kaiya II & Canino superior & Izq. & O. flavescens & $\mathrm{macho}$ & Macho & 3,5 años & 5 años & Primavera \\
\hline
\end{tabular}

Tabla 2. Estimación de edad y estación de muerte en caninos de pinnípedos de conjuntos de la región del canal Beagle (Ref. Lat: lateralidad, izq: izquierda, der: derecha, conserv: conservación).

I, solo fue posible realizar lecturas de los anillos externos (Figura 2A), ya que, a pesar de que se seleccionaron aquellos dientes que externamente denotaban una buena preservación, la estructura interna se encontraba alterada (Figura 2C). De los cinco dientes estudiados -todos de la especie $A$. australis-, cuatro corresponden a machos juveniles $(83,3 \%)$ : tres de ellos superan el año de vida, y uno, los tres años. El individuo restante es un macho adulto de siete años (Tabla 2).

En Ajej I, el ancho de la corona de los caninos analizados de $A$. australis indica que cuatro son hembras $(66,7 \%)$, tres subadultas (entre cuatro y seis años) (Figura 2B) y una adulta (siete años). Los otros dos individuos corresponden a machos subadultos (entre cuatro y cinco años). Respecto de la estación de muerte, tres murieron en verano, dos en otoño, y en el último caso, no fue posible determinarlo (Tabla 2).

En Kaiyawoteha II, de los caninos analizados, tres corresponden a machos (dos de A. australis y uno de $O$. flavescens), mientras que el cuarto individuo no pudo ser determinado ni a nivel de especie ni de sexo. En los dos individuos de la primera especie, el conteo de las líneas de dentina indica que uno corresponde a un adulto (ocho años) y el otro a un subadulto (cinco años) y que las estaciones de muerte fueron el verano y el otoño respectivamente. El espécimen de $O$. flavescens fue determinado como un macho subadulto (cinco años), muerto en primavera. El individuo indeterminado tenía una edad de seis años y la estación de muerte fue verano (Tabla 2).

\section{DISCUSIÓN}

Las estimaciones de edad a partir del análisis de cortes delgados en caninos de conjuntos de la región del canal Beagle muestran ciertas diferencias con los perfiles de mortalidad descriptos a partir de los huesos del poscráneo (Figura 3). Los trabajos previos en Imiwaia I indicaron que los adultos fueron la categoría etaria más abundante $(\mathrm{NMI} \%=53)$, seguidos por subadultos $(\mathrm{NMI} \%=44)$ y cachorros $(\mathrm{NMI} \%=3)$ (Martinoli, 2018). Por su parte, la lectura de los anillos externos (por mala conservación de la estructura interna) indica que el $80 \%$ de los individuos corresponde a subadultos, mientras que solo el $20 \%$ son individuos adultos. En el caso de Ajej I, la variabilidad es aún mayor (Figura 3). El perfil de mortalidad reconstruido a partir de los huesos del poscráneo indicó que los adultos representan el $82 \%$ de los individuos, y los subadultos, el 18\% (Martinoli y Vázquez, 2017); mientras que los cortes delgados indicaron una relación inversa: adultos $(\mathrm{NMI} \%=16,7)$ y subadultos $(\mathrm{NMl} \%=83,3)$. Por último, en Kaiyawoteha II, la diferencia entre ambos perfiles es menor (Figura 3). 


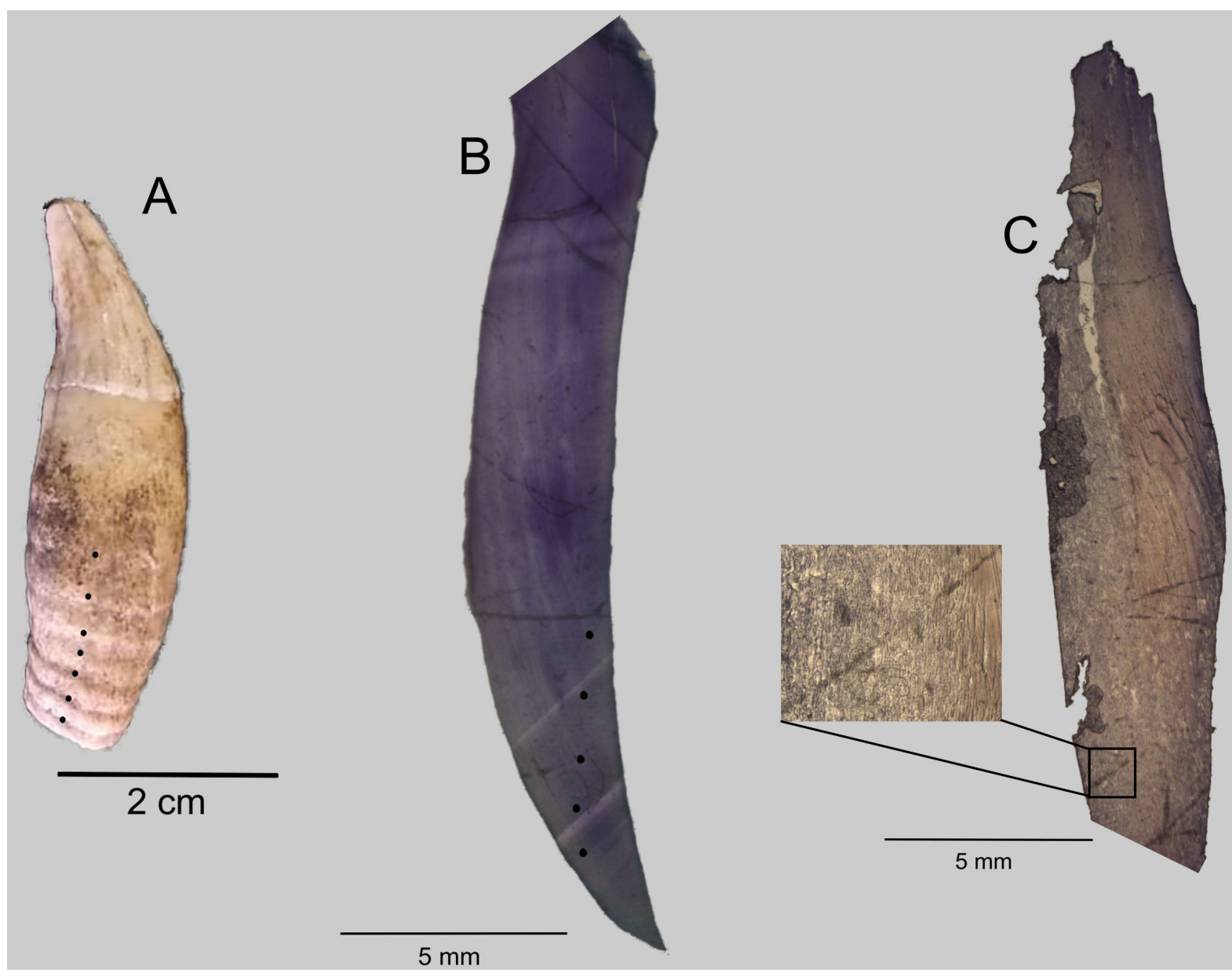

Figura 2. Caninos de pinnípedos de conjuntos de la región del canal Beagle: A) canino superior izquierdo de $A$. australis macho adulto del Segundo Componente de Imiwaia I; B) corte delgado de canino inferior derecho de hembra adulta de $A$. australis de Ajej I; C) corte delgado de canino superior derecho de macho de A. australis del Segundo Componente de Imiwaia I, con detalle del estado de conservación.

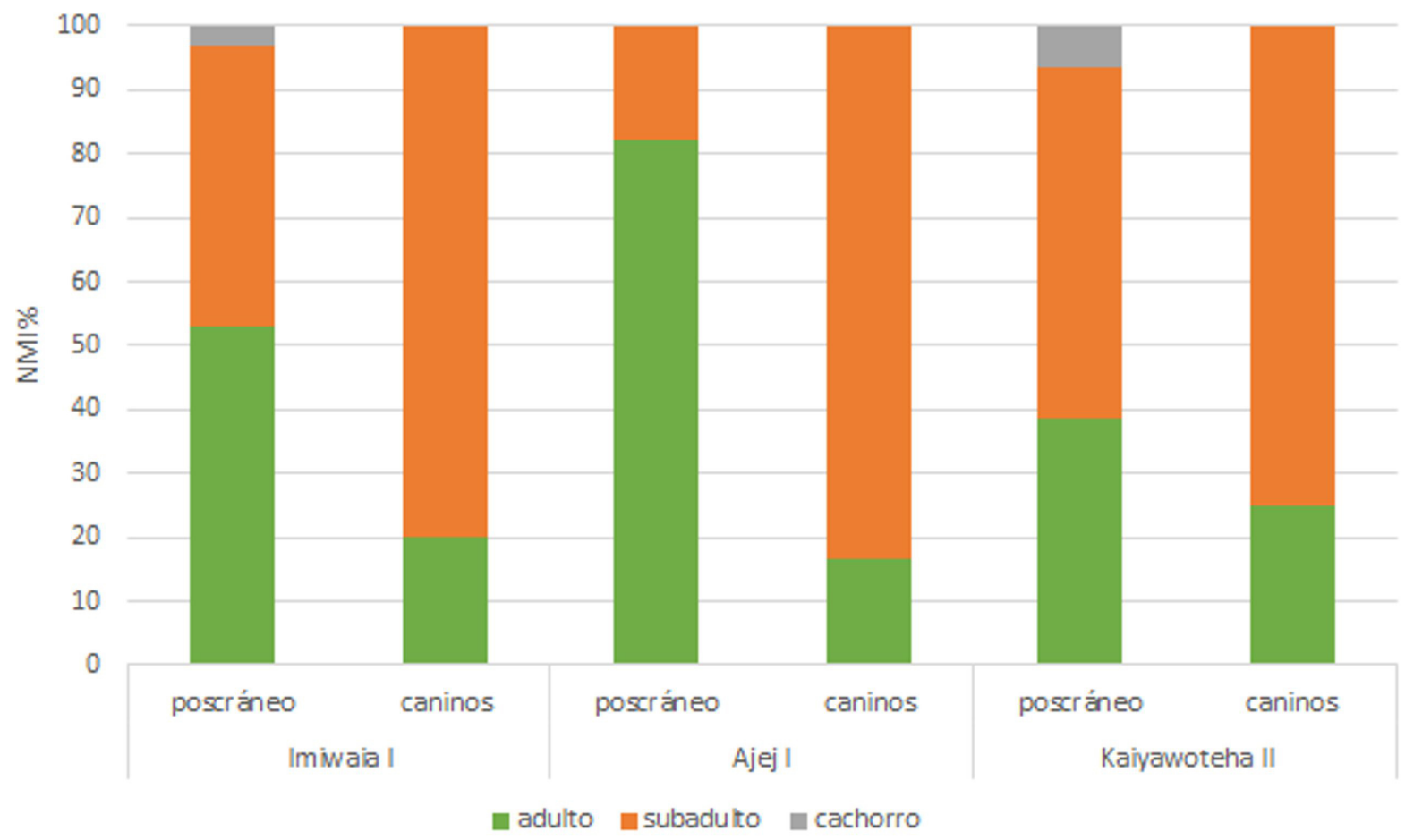

Figura 3. Histograma comparativo de las categorías etarias determinadas a partir de huesos del poscráneo (Martinoli, 2018) y de los caninos. 
Los huesos poscraneales señalaron que los adultos son el 38,7\% de los individuos; los subadultos, el 54,8\%; y los cachorros, el 6,5\% (Martinoli, 2018). Por su parte, los cortes delgados muestran que los adultos representan el $25 \%$ de los individuos; los subadultos, el $75 \%$; y no se hallaron caninos correspondientes a individuos menores a un año. Hay dos aspectos a ser destacados con respecto a esta información. Por un lado, la muestra sobre la cual se estudiaron las estructuras de crecimiento (internas y externas) parte de un NMI mucho menor que el correspondiente a los elementos poscraneales analizados en trabajos previos, lo cual implica que los resultados aquí presentados tienen una representatividad parcial del conjunto zooarqueológico. Por otro lado, los ritmos de fusión esqueletaria en ambas especies son largos y disímiles entre porciones y solo se cuenta con un esquema preliminar (Borella et al., 2013).

En segundo lugar, en lo que respecta a la estación de muerte, solo pudo ser determinada para el Holoceno tardío, y los resultados indican que los pinnípedos capturados murieron en distintos momentos del año, aunque en ningún caso se haIlaron individuos muertos en invierno. Del total de los dientes analizados $(\mathrm{N}=9)$, cinco $(55,5 \%)$ corresponden a individuos que murieron en verano, tres $(33,3 \%)$ en otoño y uno $(11,2 \%)$ en primavera. Es interesante el registro de tres hembras en edad reproductiva (más de cuatro años) de $A$. australis en Ajej I cuyas muertes ocurrieron en verano. Se espera que este tipo de ejemplares se presenten en colonias reproductivas para este momento del año, las cuales actualmente se encuentran emplazadas en sectores exteriores del archipiélago fueguino. No obstante, se observan algunas pequeñas colonias de descanso de esta especie (con hembras adultas e individuos inmaduros) a partir del mes de febrero en algunos islotes del canal Beagle (Milano et al., 2020).

La estación de muerte relevada en Ajej I y en Kaiyawoteha II contrasta con los resultados obtenidos previamente por Schiavini (1990) en el Segundo Componente de Túnel I. En este último conjunto se identificó el predominio de machos adultos y subadultos muertos principalmente en invierno, lo que se interpretó como una pauta centrada en la captura de ejemplares que se distribuían en el sector de canales fuera del período y los espacios reproductivos. Sin embargo, los conjuntos del Holoceno tardío aquí analizados indican una estacionalidad distinta. Aún si consideramos la estación de muerte informada por Schiavini (1990) para otros conjuntos del Holoceno tardío de la región, no es posible señalar un patrón de captura estacional. Como se observa en la Figura 4A, las muertes parecen haber ocurrido a lo largo de todo el año, e incluso los aprovisionamientos realizados en verano parecen aumentar entre los 2000 y los 1000 años AP. Por lo tanto, las estaciones de muerte identificadas muestran mayor variabilidad a la observada en el Segundo Componente de Túnel I. Si bien estos conjuntos no proporcionan un tamaño de muestra tan elevado como el del Segundo Componente de Túnel I (NMI = 61), sí parten de una diversidad mayor de sitios con diferentes localizaciones en la región y corresponden a distintos momentos de la secuencia de ocupación. Asimismo, si comparamos los perfiles de mortalidad determinados previamente a partir de los huesos del poscráneo en distintos conjuntos de la región del canal Beagle (Tabla 3), es posible también observar variabilidad, especialmente en la presencia de individuos menores a un año $y$ de hembras (Figura 4B). Por esto, considerando la evidencia previa, en conjunto con la información de estacionalidad presentada en este trabajo, parece haber una mayor heterogeneidad en las estrategias de aprovisionamiento con respecto a la propuesta para el Holoceno medio (Zangrando et al., 2014; Martinoli y Vázquez, 2017; Martinoli, 2018; Martinoli y Zangrando, 2021).

Ahora bien, la heterogeneidad observada en las estaciones de muerte relevadas parece general en el Holoceno tardío, es decir, con posterioridad a la ocupación del Segundo Componente de Túnel; por lo cual, cabe preguntarse a qué aspecto corresponde el patrón de capturas concentradas en invierno relevadas en dicho sitio. No obstante, al no ser posible determinar la estacionalidad de las capturas en Imiwaia I (Holoceno medio), no estamos en condiciones de afirmar si lo observado responde a un factor situacional y/o temporal. En otro sentido, el aumento de capturas de pinnípedos (especialmente de hembras en edad reproductiva) ocurridas en verano podrían potencialmente relacionarse con los cambios en la subsistencia identificados en la costa norte del canal Beagle a lo largo del Holoceno en el marco del poblamiento de la región (Zangrando, 2009; Tivoli y Zangrando, 2011). Previamente se propuso que a lo largo del Holoceno ocurrieron dos procesos (Zangrando, 2009): a) uno de diversificación (5000 años AP), el que implicó un aumento en el consumo de presas alternativas a los pinnípedos 
(guanacos y aves); es decir, comenzaron a explotarse nuevos espacios para el aprovisionamiento de recursos; y b) uno de intensificación (1500 años $A P)$, identificado a partir de un aumento en el consumo de presas pequeñas (peces) de espacios pelágicos; es decir, una ampliación en los rangos de aprovisionamiento de recursos y un aumento en la inversión laboral. En consecuencia, la mayor presencia de presas capturadas en verano en el Holoceno tardío podría indicar la explotación de espacios reproductivos y/o de descanso disponibles en distintos tramos del canal Beagle, como parte de la incorporación de nuevos espacios de aprovisionamiento.

\section{CONCLUSIÓN}
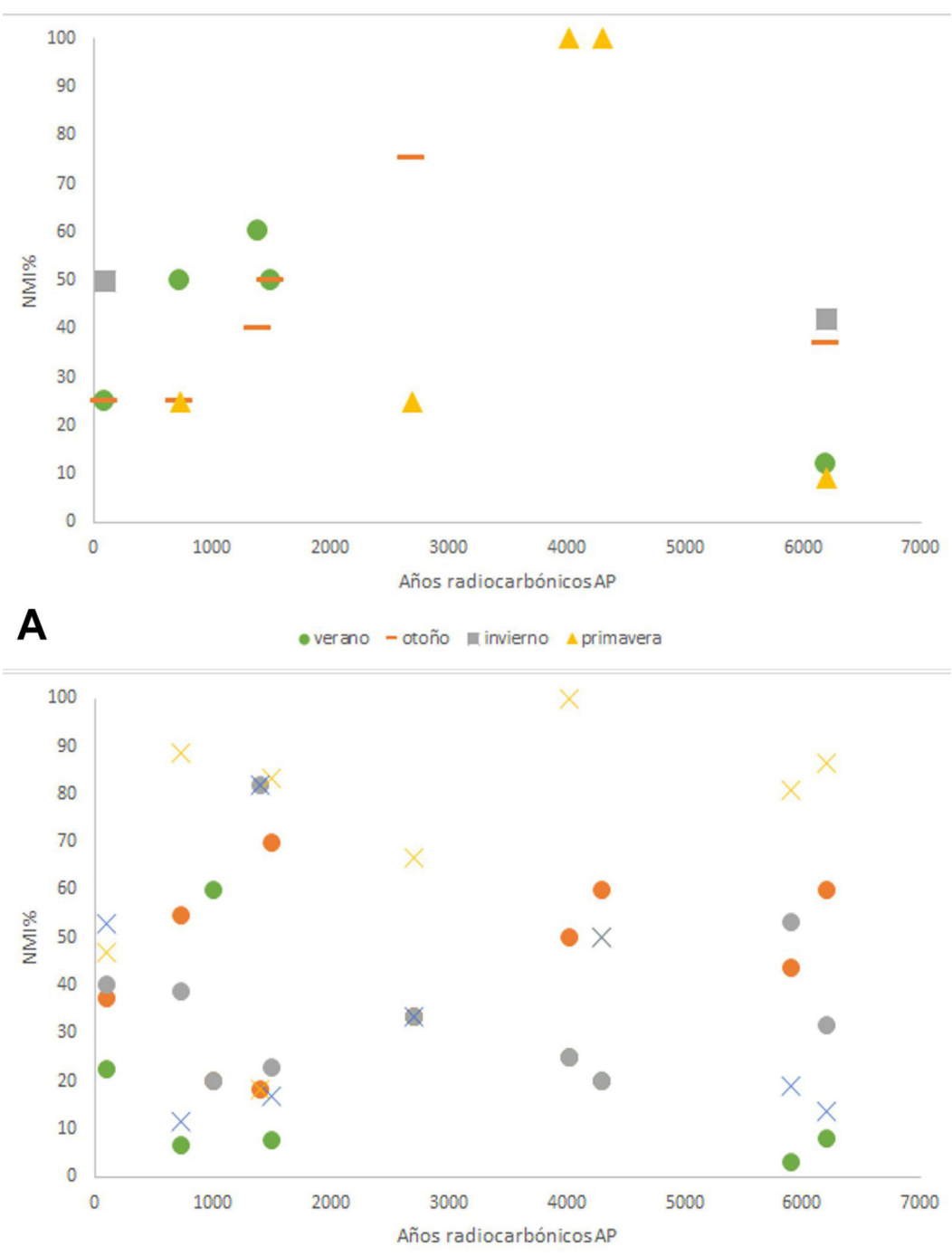

B - cachorros esubadutos adultos x machos xhembras

Figura 4. Gráficos de dispersión que indican relación temporal entre: A) estación de muerte. Los datos utilizados surgen a partir de la integración de toda la información disponible respecto de perfiles de mortalidad y estación de muerte (referencias en la Tabla 3); B) perfiles de edad y sexo.
El estudio de 15 caninos correspondientes a pinnípedos capturados en la región del canal Beagle a lo largo del Holoceno nos permite resaltar varios aspectos. En primer lugar, fue posible hallar cierta discrepancia entre las lecturas de edad realizadas a partir de cortes delgados descalcificados y teñidos y los perfiles de mortalidad propuestos en función del esqueleto poscraneal. Lo dicho podría relacionarse tanto con el tamaño de la muestra aquí estudiada, así como con el esquema de fusión preliminar, que puede quedar incompleto dados los ritmos de fusión disímiles en los otáridos analizados.

En segundo lugar, según los resultados obtenidos en este trabajo para el Holoceno tardío, se ha podido determinar que más del $50 \%$ de los casos fueron individuos capturados en verano y que algunos de ellos corresponden a hembras en edad reproductiva. Asimismo, al comparar los datos aquí presentados con información de estacionalidad generada para otros conjuntos de la región con diferentes cronologías, es posible observar que los pinnípedos fueron capturados en distintos momentos del año, incluido el verano. Lo dicho implica, por un lado, que los individuos de A. australis pueden haber estado disponibles en aguas interiores del canal Beagle en época reproductiva, al menos temporalmente, según cambios ambientales de corto plazo (Saporiti et al., 2014; Martinoli, 2018). Por otro lado, esto contrasta con la información previa obtenida en el Segundo Componente de Túnel I (Holoceno medio), según la cual los lobos marinos fueron capturados mayormente en invierno. Por este motivo, el modelo de subsidio energético y estrategia conservadora del recurso, al explotar indirectamente presas propias de otros espacios con una productividad mayor (Schiavini, 1990), se ajustaría mejor a conjuntos del Holoceno medio, en tanto los perfiles de mortalidad y la estación de muerte identificados para conjuntos más recientes indican condiciones más diversas 


\begin{tabular}{|c|c|c|c|c|c|c|}
\hline \multicolumn{2}{|l|}{ Conjuntos } & Túnel I (2do) & $\begin{array}{l}\text { Imiwaia I } \\
\text { (2do) }\end{array}$ & Túnel I (3er) & $\begin{array}{l}\text { L. Packewaia } \\
\text { (Antiguo) }\end{array}$ & Túnel I (4to) \\
\hline \multicolumn{2}{|l|}{ Referencias } & \begin{tabular}{|l|}
$\begin{array}{l}\text { Schiavini } \\
(1990)\end{array}$ \\
\end{tabular} & \begin{tabular}{|l|}
$\begin{array}{l}\text { Martinoli } \\
(2015)\end{array}$ \\
\end{tabular} & \begin{tabular}{|l}
$\begin{array}{l}\text { Martinoli } \\
(2018)\end{array}$ \\
\end{tabular} & Saxon (1979) & Martinoli (2018) \\
\hline \multicolumn{2}{|c|}{ Cronología $\left({ }^{14} \mathrm{C}\right.$ años $\left.\mathrm{AP}\right)$} & $6200-4500$ & $5900-5700$ & 4300 & 4020 & 2700 \\
\hline \multirow{3}{*}{ Edad } & Cachorros & 18 & 1 & 1 & 4 & 2 \\
\hline & Subadultos & 134 & 14 & 3 & 8 & 2 \\
\hline & Adultos & 71 & 17 & 1 & 4 & 2 \\
\hline \multirow{2}{*}{ Sexo } & Machos & 193 & 17 & 1 & 1 & 2 \\
\hline & Hembras & 30 & 4 & 1 & 0 & 1 \\
\hline \multirow{4}{*}{$\begin{array}{l}\text { Estación de } \\
\text { muerte }\end{array}$} & Verano & 5 & - & 0 & 0 & 0 \\
\hline & Otoño & 15 & - & 0 & 0 & 3 \\
\hline & Invierno & 17 & - & 0 & 0 & 0 \\
\hline & Primavera & 4 & - & 3 & 1 & 1 \\
\hline \multicolumn{2}{|l|}{ Conjuntos } & \begin{tabular}{|l}
$\begin{array}{l}\text { L. Packewaia } \\
\text { (Reciente) }\end{array}$ \\
\end{tabular} & Ajej I & Shamakush I & $\begin{array}{l}\text { Kaiyawoteha } \\
\text { II }\end{array}$ & Túnel VII \\
\hline \multicolumn{2}{|l|}{ Referencias } & Saxon (1979) & \begin{tabular}{|l|}
$\begin{array}{l}\text { Martinoli } \\
(2018)\end{array}$ \\
\end{tabular} & \begin{tabular}{|l|} 
Orquera y \\
Piana (1996)
\end{tabular} & \begin{tabular}{|l|}
$\begin{array}{l}\text { Martinoli } \\
(2018)\end{array}$ \\
\end{tabular} & \begin{tabular}{|l} 
Zangrando et al. \\
$(2010)$
\end{tabular} \\
\hline \multicolumn{2}{|c|}{ Cronología $\left({ }^{14} \mathrm{C}\right.$ años $\left.\mathrm{AP}\right)$} & $1500-280$ & 1400-1200 & 1000 & 730 & 100 \\
\hline \multirow{3}{*}{ Edad } & Cachorros & 5 & 0 & 3 & 2 & 9 \\
\hline & Subadultos & 46 & 2 & 1 & 17 & 15 \\
\hline & Adultos & 15 & 9 & 1 & 12 & 16 \\
\hline \multirow{2}{*}{ Sexo } & Machos & 15 & 2 & - & 23 & 8 \\
\hline & Hembras & 3 & 9 & - & 3 & 9 \\
\hline \multirow{4}{*}{$\begin{array}{l}\text { Estación de } \\
\text { muerte }\end{array}$} & \begin{tabular}{|l|} 
Verano \\
\end{tabular} & 9 & 3 & - & 2 & 1 \\
\hline & Otoño & 9 & 2 & - & 1 & 1 \\
\hline & \begin{tabular}{|l|} 
Invierno \\
\end{tabular} & 0 & 0 & - & 0 & 2 \\
\hline & Primavera & 0 & 0 & - & 1 & 0 \\
\hline
\end{tabular}

Tabla 3. Perfiles de edad, sexo y estación de muerte de conjuntos de pinnípedos (NMI) de la región del canal Beagle con cronologías del Holoceno.

en la captura de pinnípedos.

Por último, cabe la posibilidad de que el aumento de capturas de pinnípedos en distintos momentos del año indique potencialmente que los grupos humanos que habitaron el tramo central de la costa sur de Tierra del Fuego comenzaron a incorporar nuevos espacios de aprovisionamiento como parte del propio proceso de poblamiento en la región (Zangrando, 2009).

\section{Agradecimientos}

Este trabajo se ha desarrollado en el marco del PICT 1230-2017. Agradecemos a los integrantes del Laboratorio de Mamíferos Marinos del CESIMAR - CENPAT- CONICET, por su colaboración. Agradecemos a los revisores anónimos por las valiosas sugerencias realizadas.

\section{REFERENCIAS CITADAS}

Ambrústolo, P., Zubimendi, M. y Crespo E. (2019).

Death seasonality determination from pinnipeds teeth: the Cueva del Negro site case (Argentine Patagonia). Arqueología, 25(2), 247-262.

Borella, F. (2006). ¿Dónde están los lobos en la costa atlántica de norpatagonia? Explorando vías para resolver el registro arqueofaunístico. Werkén, 9, 97-114.

Borella, F. (2016). Antes del Faro. La explotación de mamíferos marinos en la localidad arqueológica Faro San Matías durante el Holoceno tardío (Norpatagonia, Argentina). En F. Mena (Ed.), Arqueología de Patagonia: De mar a mar (pp. 295-304). Ediciones CIEP/Ñire Negro.

Borella, F., Grandi, M., Vales, D., Goodall, R. y Crespo, E. (2013). Esquema preliminar de fusión epifisaria en huesos de lobos marinos (Arctocephalus australis y Otaria flavescens), su contribución en los análisis zooarqueológicos. En F. Zangrando, R. Barberena, A. Gil, G. Neme, M. Giardina, L. Luna, C. Otaola, S. Paulides, L. Salgán y A. Tivoli (Eds.), Tendencias 
teórico-metodológicas y casos de estudio en la arqueología de Patagonia (pp. 39-51). Museo de Historia Natural.

Borella, F., Vales, D., Grandi, M. y García, N. (2018). Rasgos diagnósticos en elementos postcraneales de dos especies de otáridos para su identificación en el registro zooarqueológico. Magallania, 46(2), 187-203.

Cadegán Sepúlveda, K. (2013). Anatomía comparada del esqueleto apendicular de dos especies de otáridos, Otaria flavescens (Shaw, 1800) Arctophoca australis gracilis (Zimmerman, 1783) [tesis de grado. Universidad Austral de Chile].

Christensen, M. (2016). La industria ósea de los cazadoresrecolectores: El caso de los nómadas marinos de Patagonia y Tierra del Fuego. Ediciones Universidad de Magallanes.

Crespo, E. (1984). Dimorfismo sexual en los dientes caninos y en los cráneos de lobo marino del sur, Otaria flavescens (Shaw) (Pinnipedia, Otariidae). Revista del Museo Argentino de Ciencias Naturales (Bernardino Rivadavia) e Instituto Nacional de Investigación de las Ciencias Naturales, 13, 245-254.

Crespo, E. (1988). Dinámica poblacional del lobo marino de un pelo Otaria flavescens (Shaw, 1800), en el norte del litoral patagónico [tesis doctoral. Universidad de Buenos Aires].

Crespo, E., Schiavini, A., Pérez Macri, G., Reyes, L. y Dans, S. (1994). Estudios sobre determinación de edad de mamíferos marinos del atlántico sudoccidental. En J. Oporto (Ed.), Anales de la Cuarta Reunión de Trabajo de Especialistas en Mamíferos Marinos Acuáticos de América del Sur (pp. 31-55). Centro de Investigaciones y Manejo de Mamíferos Marinos.

Estévez, J. y Martínez, J. (1995). Reflexiones metodológicas a partir del análisis de los restos de pinnípedos de Túnel VII. En J. Estévez Escalera y A. Vila Mitjá (Eds.), Encuentros en los conchales fueguinos (pp. 1831933). Treballs d’Etnoarquelogía. Consejo Superior de Investigaciones Científicas, Universidad Autónoma de Barcelona.

Grayson, D. (1984). Quantitative Zooarchaeology. Academic Press.

Hohn, A., Scott, M., Wells, R., Sweeney, J. e Irvine, A. (1989). Growth layers in teeth from known-age, freeranging bottlenose dolphins. Marine Mammal Science, 5, 315-342.

Legoupil, D. (1989). La identificación de los mamíferos marinos en los sitios canoeros de Patagonia: problema y constataciones. Anales del Instituto de la Patagonia, 19, 101-113.

Loza, C., Soibelzon, L., Tarnawski, B., del Corro, M., Negrete, J. y Ciancio, M. (2016). Determinación de edades en Otariidae y Phocidae (Carnivora) sobre piezas dentarias, técnicas alternativas. Revista del Museo de La Plata, 1(1), 39-56.

Lyman, R. L. (1988). Zoogeography of Oregon coast marine mammals: the last 3,000 years. Marine Mammal Science, 4(3), 247-264.

Lyman, R. L. (1989). Seal and Sea-lion Hunting: A Zooarchaeological Study from the Southern Northwest Coast of North America. Journal of Anthropological Archaeology, 8, 68-99.

Lyman, R. L. (1994). Vertebrate Taphonomy. Cambridge University Press.

Lyman, R. L. (2003). Pinniped Behavior, Foraging Theory, and the Depression of Metapopulation and Nondepression of a Local Population on the Southern Northwest Coast of North America. Journal of Anthropological Archaeology, 22(4), 376-388.

Martinoli, M. P. (2013). Modalidades de explotación de pinnípedos en las ocupaciones canoeras tempranas del canal Beagle: el sitio Imiwaia I (Tierra del Fuego, Argentina) [tesis de grado. Universidad de Buenos Aires].

Martinoli, M. P. (2015). Procesamiento y consumo de pinnípedos: el caso de las ocupaciones canoeras tempranas del sitio Imiwaia I (Tierra del Fuego, República Argentina). Intersecciones en Antropología, 16(2), 367-381.

Martinoli, M. P. (2017). Pautas de procesamiento y consumo de pinnípedos en la costa sur de Tierra del Fuego e Isla de los Estados: un análisis comparativo. Arqueología, 23(3), 173-196.

Martinoli, M. P. (2018). Modalidades de explotación, procesamiento y consumo de pinnípedos en la margen meridional de Tierra del Fuego [tesis doctoral. Universidad de Buenos Aires].

Martinoli, M. P. y Vázquez, M. (2017). Pinniped Capture and Processing: a Comparative Analysis from Beagle Channel (Tierra del Fuego, Argentina). En M. Mondini, S. Muñoz y P. Fernández (Eds.), Zooarchaeology in the Neotropics: Environmental Diversity and HumanAnimal Interactions (pp. 7-23). Springer.

Martinoli, M. P. y Zangrando, A. F. (2021). Variabilidad en la explotación de pinnípedos: el caso de la margen meridional de Tierra del Fuego. Latin American Antiquity, aceptado con modificaciones.

Milano, V. N., Grandi, M., Schiavini, A. y Crespo, E. (2020). Recovery of South American fur seals from Fuegian Archipelago (Argentina). Marine Mammal Science, 36, 1022-1032. 10.1111/mms.12686

Molina-Schiller, D. y Pinedo, M. (2004). Growth layer patterns in Arctocephalus australis canine teeth: 
evaluation of techniques for age determination. Latin American Journal of Aquatic Mammals, 3(2), 107-118.

Muñoz, S. (2011). Pinniped Zooarchaeological Studies in Southern Patagonia: Current Issues and Future Research Agenda. En N. Bicho, J. Haws y L. Davis (Eds.), Trekking the Shore. Changing Coastlines and the Antiquity of Coastal Settlement (pp. 305-331). Springer.

Myrick, A., Hohn, A., Sloan, P., Kimura, M. y Stanley, D. (1983). Estimating the age of spotted and spinner dolphins (Stenella attenuata and Stenella longirostris) from teeth. National Oceanic Atmospheric Administration Technical Memorandum NMFS, SWFC30. $17 \mathrm{pp}$.

Newsome, S., Clementz, M. y Koch, P. (2010). Using stable isotope biogeochemistry to study marine mammal ecology. Marine Mammal Science, 26(3), 509-572.

Nye, J., Zangrando, A. F., Martinoli, M. y Fogel, M. (2020). Temporal and population trends in human exploited pinnipeds from Tierra del Fuego. Palaeogeography, Palaeoclimatology, Palaeoecology, 554. 10.1016/j. palaeo.2020.109804

Orquera, L. y Piana, E. (1987). Human littoral adaptation in the Beagle Channel region: the maximum possible age. Quaternary of South America and Antarctic Peninsula, 5, 133-65.

Orquera, L. y Piana, E. (1995). Lancha Packewaia: actualización y rectificaciones. Relaciones de la Sociedad Argentina de Antropología, 19, 325-362.

Orquera, L. y Piana, E. (1996). El sitio Shamakush I (Tierra del Fuego, República Argentina). Relaciones de la Sociedad Argentina de Antropología, 21, 215-265.

Orquera, L. y Piana, E. (1999). Arqueología de la región del canal Beagle (Tierra del Fuego, Argentina). Sociedad Argentina de Antropología.

Orquera, L. y Piana, E. (2009). Sea Nomads of the Beagle Channel in Southernmost South America: Over Six Thousand Years of Coastal Adaptation and Stability. Journal of Island \& Coastal Archaeology, 4, 61-81.

Pérez García, M. (2003). Osteología comparada del esqueleto postcraneano de dos géneros de otariidae del Uruguay. Boletín de la Sociedad Zoológica del Uruguay, 14, 1-16.

Pérez García, M. (2008). Ontogenia del postcráneo de Arctocephalus australis (mammalia, carnivore, otariidae). Boletín de la Sociedad Zoológica del Uruguay 17, 1-19.

Perrin, W. y Myrick, A. (1980). Growth of odontocetes and sirenians: problems in age determination. Reports of the International Whaling Commission (Special Issue 3).

Piana, E., Vázquez, M. y Álvarez, M. (2008). Nuevos resultados del estudio del sitio Ajej I: un aporte a la variabilidad de estrategias de los canoeros fueguinos. Runa, 29, 101-121.

Piana, E., Vázquez, M. y Ceraso, A. (2007). Kaiyawoteha. Informe de excavación. Informe preparado a solicitud de LOS PETRELES S.A. para cumplimentar con el Análisis de Impacto al Patrimonio Cultural Provincial, Ley Provincial N³70.

Rosas, F., Haimovici, M. y Pinedo, M. (1993). Age and growth of the South American sea lion, Otaria flavescens (Shaw, 1800), in southern Brazil. Journal of Mammalogy, 74(1), 141-147.

Sanfelice, D. y Ferigolo, J. (2008). Estudo comparativo entre os sincrânios de Otaria byronia e Arctocephalus australis (Pinnipedia, Otariidae). Iheringia. Série Zoología, 98(1), 5-16.

Saporiti, F., Bala, L., Gómez Otero, J., Crespo, E., Piana, E., Aguilar, A. y Cardona, L. (2014). Paleoindian pinniped exploitation in South America was driven by oceanic productivity. Quaternary International, 352, 85-91.

Saxon, E. (1979). Natural Prehistory: the archaeology of Fuego-Patagonian ecology. Quaternaria, 21, 329-356.

Scheinsohn, V. 1997. Explotación de materias primas óseas en la Isla Grande de Tierra del Fuego [tesis doctoral. Universidad de Buenos Aires].

Scheinsohn, V. (2010). Hearts and Bones. Bone Raw Material Exploitation in Tierra del Fuego. BAR International Series 2094. Archaeopress.

Scheinsohn, V. y Ferretti, J. L. (1995). The mechanical properties of bone materials in relation to the design and function of prehistoric tools from Tierra del Fuego, Argentina. Journal of Archaeological Science, 22(6), 711-717.

Schiavini, A. (1990). Estudio de la relación entre el hombre y los pinnípedos en el proceso adaptativo humano del canal Beagle (Tierra del Fuego, Argentina) [tesis doctoral. Universidad de Buenos Aires].

Schiavini, A. (1993). Los lobos marinos como recursos para cazadores-recolectores marinos: el caso de Tierra del Fuego. Latin American Antiquity, 4, 346-366.

Schiavini, A., Crespo, E. y Szapkievich, V. (2004). Status of the population of South American sea lion (Otaria flavescens Shaw, 1800) in southern Argentina. Mammalian Biology-Zeitschrift für Säugetierkunde, 69(2), 108-118.

Schiavini, A., Lima, M. y Batalles, L. (1992). Growth structures of maxillary canines of the southern fur seal (Arctocephalus australis). Marine Mammal Science, 8(1), 89-93.

Schiavini, A. y Raya Rey, A. (2001). Aves y Mamíferos Marinos en Tierra del Fuego. Estado de 
Nuevos datos de estimación de edad y estación de muerte de pinnípedos en la región del canal... Intersecciones en Antropología 22(2), julio-diciembre. 2021. ISSN-e 1850-373X

situación, interacción con actividades humanas y recomendaciones para su manejo. Informe preparado bajo contrato con el proyecto "Consolidación e Implementación del Plan de Manejo de la Zona costera Patagónica", proyecto ARG/97/G31 GEF/PNUD/ MRECIC CADIC-CONICET.

Tivoli, A. y Zangrando, A. F. (2011). Subsistence variations and landscape use among maritime hunter-gatherers. A zooarchaeological analysis from the Beagle Channel (Tierra del Fuego, Argentina). Journal of Archaeological Science, 38(5), 1148-1156.

Vales, D. G., Cardona, L., Zangrando, A. F., Borella, F., Saporiti, F., Goodall, R., Oliveira, L. y Crespo, E. (2017). Holocene changes in the trophic ecology of an apex marine predator in the South Atlantic Ocean. Oecologia, 183(2), 555-570.

Zangrando, A. F. (2009). Historia evolutiva y subsistencia de cazadores-recolectores marítimos de Tierra del Fuego. Sociedad Argentina de Antropología.

Zangrando, A. F. (2014). Human Predation on Pinnipeds in the Beagle Channel. En S. Muñoz, C. Götz y
E. Ramos Roca (Eds.), Neotropical and Caribbean Aquatic Mammals. Perspectives from Archaeology and Conservation Biology (pp. 161-174). Nova Publishers.

Zangrando, A. F., Orquera, L. y Piana, E. (2010).

Diversificación e intensificación de recursos animales en la secuencia arqueológica del canal Beagle (Tierra del Fuego, Argentina). En M. Gutiérrez, M. De Nigris, P. Fernández, M. Giardina, A. Gil, A. Izeta, G. Neme y H. Yacobaccio (Eds.) Zooarqueología a principios del Siglo XXI: Aportes teóricos, metodológicos y casos de estudio (pp. 359-370). Ediciones del Espinillo.

Zangrando, F., Panarello, H. y Piana, E. (2014).

Zooarchaeological and stable isotopic assessments on pinniped-human relations in the Beagle Channel (Tierra del Fuego, southern South America). International Journal of Osteoarchaeology, 24(2), 231-244. 
